\section{miRWalk2.0: a comprehensive atlas of microRNA-target interactions}

To the Editor: miRWalk2.0 (http://zmf.umm.uni-heidelberg. $\mathrm{de} /$ mirwalk2) is a comprehensive archive, supplying the largest available collection of predicted and experimentally verified microRNA (miRNA)-target interactions ( 949 million), with novel and unique features that improve upon the previous version miRWalk ${ }^{1}$. miRWalk2.0 was developed to generate possible miRNA interactions with all the regions of a gene by gathering 13 prediction data sets from existing miRNA-target resources (Table 1, Supplementary Methods, Supplementary Fig. 1 and Supplementary Tables 1-3). The web interface of miRWalk2.0, which contains a predicted-target module and a validated-target module, hosts a multilayered view of data and offering information on miRNAs, genes, epigenomics, pathways, ontologies, protein classes, phenotype, genotype, single-nucleotide polymorphisms, functional networks, tandem mass spectra and relevant PubMed articles. Additionally, information on human homologous genes across 15 species can be downloaded to conduct an interspecies analysis. Notably, lists of genes or their identifiers can also be created for external tools such as the Database for Annotation, Visualization and Integrated Discovery (DAVID) ${ }^{2}$.

Table 1 | Overview of information documented in miRWalk2.0

\begin{tabular}{|c|c|c|c|c|}
\hline Category & Human & Mouse & Rat & $\begin{array}{l}\text { All analyzed } \\
\text { species }\end{array}$ \\
\hline Genes & 20,022 & 22,232 & 22,817 & $308,700^{\mathrm{a}}$ \\
\hline $\begin{array}{l}\text { Noncoding } \\
\text { RNAs (miRNAs } \\
\text { and IncRNAs) }\end{array}$ & 30,246 & 11,869 & 31,559 & 80,208 \\
\hline Identifiers & $1,021,803$ & 694,514 & 395,099 & $5,146,217^{a}$ \\
\hline $\begin{array}{l}\text { Pathways } \\
\text { (KEGG, Wiki, } \\
\text { Panther) }\end{array}$ & 590 & 492 & 493 & $6,234^{\mathrm{a}}$ \\
\hline $\begin{array}{l}\text { Ontologies } \\
\text { (GOs, DOs, } \\
\text { HPOs) }\end{array}$ & 16,268 & 5,441 & 5,447 & $57,797^{a}$ \\
\hline $\begin{array}{l}\text { Putative } \\
\text { miRNA-target } \\
\text { interactions }\end{array}$ & $544,235,131$ & $221,188,804$ & $86,384,672$ & $847,553,399$ \\
\hline $\begin{array}{l}\text { Validated } \\
\text { miRNA-target } \\
\text { interactions }\end{array}$ & 539,533 & 128,751 & 333 & 668,617 \\
\hline
\end{tabular}

Furthermore, miRWalk2.0 offers a framework to obtain statistically significant miRNA interactions on genes associated with pathways, ontologies, classes of gene and protein, disorders and mitochondrial genomes, and to explore pathways via the Kyoto Encyclopedia of Genes and Genomes (KEGG). Beyond prediction data, it also incorporates the experimentally verified information on miRNAs linked with genes, diseases, pathways, ontologies, cell lines and organs.

To enable large-scale enrichment analysis, a novel feature named "Customized data set" is implemented within miRWalk2.0 that allows users to generate a customized list of putative targets of their miRNAs of interest from 13 different data sets.

miRNAs have been shown to base-pair with other noncoding RNAs, especially long noncoding RNAs (lncRNAs) that play crucial roles in diverse biological processes and pathologies ${ }^{3}$. We generated a comprehensive platform for a comparative overview of miRNA-lncRNA interactions. The gene- and lncRNAbased searches in miRWalk2.0 provide a combination of targets and nontargets for the queried miRNA, which will be helpful to reduce false positive targets.

Previous studies suggest that several mammalian miRNA genes are coexpressed with their host gene or neighboring genesutilizing the host or neighbors' transcriptional machinery-and promote synergistic and/or antagonistic effects on them ${ }^{4}$. miRWalk2.0 provides a genomic location search for genes to determine which of the miRNAs share the same or a nearby location. For example, a list of disease-specific or significant genes can be interrogated to attain miRNAs that may be expressed with the queried genes that could be involved in the genetic regulation of a specific condition. This information can be used to choose and validate other miRNAs that are located nearby or within the highly differentially regulated genes.

The current status of miRWalk2.0 is depicted in Table 1 (see also Supplementary Table 3). More annotations and additional species will be integrated to further expand this resource. The validated-target module is updated every 3 months, whereas the predicted-target module is updated twice a year.

Note: Any Supplementary Information and Source Data files are available in the online version of the paper (doi:10.1038/nmeth.3485).

COMPETING FINANCIAL INTERESTS

The authors declare no competing financial interests.

\section{Harsh Dweep \& Norbert Gretz}

Medical Research Center, Medical Faculty of Mannheim, University of Heidelberg, Mannheim, Germany.

e-mail: harsh.dweep@medma.uni-heidelberg.de

1. Dweep, H., Sticht, C., Pandey, P. \& Gretz, N. J. Biomed. Inform. 44, 839-847 (2011).

2. Mootha, V.K. et al. Nat. Genet. 34, 267-273 (2003).

3. Yoon, J.H., Abdelmohsen, K. \& Gorospe, M. Semin. Cell Dev. Biol. 34, 9-14 (2014).

4. Lutter, D., Marr, C., Krumsiek, J., Lang, E.W. \& Theis, F.J. BMC Genomics 11, 224 (2010). 